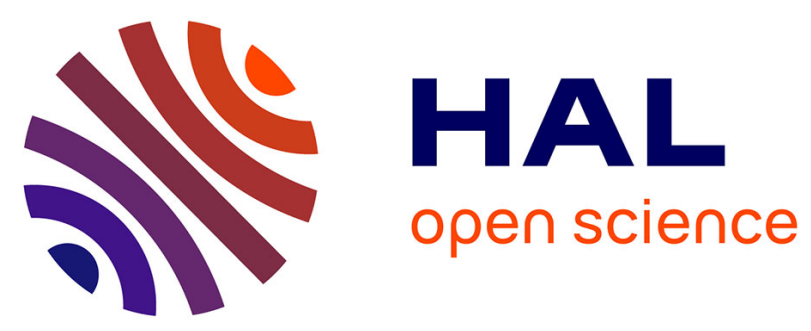

\title{
Cancer Is Not (Only) a Senescence Problem
}

Frédéric Thomas, Fabrice Vavre, Tazzio Tissot, Marion Vittecoq, Mathieu

Giraudeau, Florence F. Bernex, Dorothée Missé, François Renaud, Nynke

Raven, Christa Beckmann, et al.

\section{- To cite this version:}

Frédéric Thomas, Fabrice Vavre, Tazzio Tissot, Marion Vittecoq, Mathieu Giraudeau, et al.. Cancer Is Not (Only) a Senescence Problem. Trends in Cancer, 2018, 4 (3), pp.169 - 172. 10.1016/j.trecan.2018.01.002 . hal-01933408

\section{HAL Id: hal-01933408 https://hal.science/hal-01933408}

Submitted on 5 Dec 2018

HAL is a multi-disciplinary open access archive for the deposit and dissemination of scientific research documents, whether they are published or not. The documents may come from teaching and research institutions in France or abroad, or from public or private research centers.
L'archive ouverte pluridisciplinaire HAL, est destinée au dépôt et à la diffusion de documents scientifiques de niveau recherche, publiés ou non, émanant des établissements d'enseignement et de recherche français ou étrangers, des laboratoires publics ou privés. 


\title{
Cancer Is Not (Only) a Senescence Problem
}

\author{
Frédéric Thomas, ${ }^{1, * F a b r i c e ~ V a v r e, ~}{ }^{2}$ Tazzio Tissot, ${ }^{1,3}$ Marion Vittecoq, ${ }^{4}$ \\ Mathieu Giraudeau, ${ }^{5.6}$, Florence Bernex, ${ }^{7,8}$ Dorothée Misse, ${ }^{1}$ François Renaud, ${ }^{1}$ Nynke Raven, ${ }^{9}$ Christa \\ Beckmann,9,10Rodrigo Hamede, ${ }^{7,9}$ Peter A. Biro, ${ }^{7}$ and Beata Ujvari ${ }^{9,11}$ \\ How to cite \\ Thomas F., Vavre F., Tissot T., Vittecoq M., Giraudeau M., Bernex F., Misse D., Renaud F., Raven N., Beckmann C., Hamede R., Biro P.A., \\ Ujvari B. 2018. Cancer Is Not (Only) a Senescence Problem. Trends in Cancer 4:169-172. doi: 10.1016/j.trecan.2018.01.002
}

${ }^{1}$ CREEC/MIVEGEC, UMR IRD/CNRS/UM 5290, 911 Avenue Agropolis, BP 64501, 34394 Montpellier Cedex 5, France

${ }^{2}$ Univ Lyon, Université Lyon 1, CNRS, Laboratoire de Biométrie et Biologie Evolutive UMR5558, F-69622 Villeurbanne, France

${ }^{3}$ Institute of Ecology and Environmental Sciences - Paris, Sorbonne Université-CNRS-IRD-INRA-P7-UPEC, 4 place Jussieu, 75005 Paris, France

${ }^{4}$ Centre de Recherche de la Tour du Valat, le Sambuc, 13200 Arles, France

${ }^{5}$ Arizona State University, School of Life Sciences, Tempe, AZ 85287-4501, USA

${ }^{6}$ Centre for Ecology and Conservation, College of Life and Environmental Sciences, University of Exeter, Penryn, UK ${ }^{7}$ RHEM, IRCM, Institute of Cancer Research Montpellier, INSERM, Montpellier, France

${ }^{8}$ ICM Regional Cancer Institute of Montpellier, Montpellier, France

${ }^{9}$ Centre for Integrative Ecology, School of Life and Environmental Sciences, Deakin University, Waurn Ponds, VIC 3216, Australia

${ }^{10}$ Centre for Behavioural and Physiological Ecology, Zoology, School of Environmental and Rural Science, University of New England, Armidale, NSW 2351, Australia

${ }^{11}$ School of Biological Sciences, University of Tasmania, Private Bag 55, Hobart, TAS 7001, Australia

Age is one of the strongest predictors of cancer and risk of death from cancer. Cancer is therefore generally viewed as a senescence-related malady. However, cancer also exists at subclinical levels in humans and other animals, but its earlier effects on the body are poorly known by comparison. We argue here that cancer is a significant but ignored burden on the body and is likely to be a strong selective force from early during the lifetime of an organism. It is time to adopt this novel view of malignant pathologies to improve our understanding of the ways in which oncogenic phenomena influence the ecology and evolution of animals long before their negative impacts become evident and fatal.

Cancer defines a family of potentially lethal diseases occurring when host cells lose their normal cooperative behavior, proliferate at greater rates than would normal cells, spread, and hence become malignant. In most cases (i.e., $90 \%$ in humans and domestic animals [1]), deaths due to cancer are not attributed to locally confined tumors but rather to metastases (i.e., disseminated tumor spread). Advancing age being indisputably the most significant risk factor (in terms of incidence) for the development of metastatic cancer, the generally accepted concept is that cancer is a form of agedependent, often senescent, pathology [2]. This view is valid in several cases when, for example, aging predisposes cells to accumulate oncogenic mutations. The role of senescence on malignant progression can, however, also be indirect; that is, cancer is not per se a senescence phenomenon but a byproduct of the organism's senescence. For instance, according to Rozhok and DeGregori [3] carcinogenesis should be viewed as a function of physiological aging whereby aging and the resulting altered tissue microenvironments lead to selection on previously accumulated random mutations, some of which gain a fitness advantage. Invasive cancers can also indirectly result from the senescence of the mechanisms that normally hold in situ tumors in check (e.g., the progressive decline of the immune system with age, also termed immunosenescence).

Senescence has different meanings in different realms of study. Cell biologists usually use senescence to refer to the loss of cellular proliferative potential. Clinical experts use it to refer to age-related deterioration. Even in evolutionary biology, senescence can be defined broadly or narrowly. Besides the more-or-less direct links with senescence-associated pro-cesses (sensu deterioration that occurs in old age), cancer also displays a range of characteristics that are not found in classical agerelated diseases, suggesting that malignancies should not be simply assimilated under the umbrella of senescence. This is not a semantic problem but rather an important issue,

particularly in ecology and evolution, where late-onset diseases are frequently overlooked compared with those occurring earlier in life, as they have limited effect on the evolutionary trajectory of species due to their impact manifesting only post- 
reproduction. Thus, mistakenly viewing cancer as a senescence disease leads to a potential under-appreciation of its ecological and evolutionary importance.

There is a long list of cancer attributes that should motivate scientists to consider cancer as a disease differing from a 'senescence problem'. The first obvious attribute is that cancer ironically relies on the bypassing of cellular senescence. A second attribute is that, although rare, several forms of cancer are not restricted to occurring only in the elderly but also develop from early childhood and/or in young adults (e.g., gliomas, leukemia, testicular cancer). In addition, accumulated mutational damage from environ-mental exposures does not qualify as senescence. For example, the scenario of a 5-yearold person with high UV-light exposure developing cancer at age 8 years would not be classified as senescence. It is therefore important to distinguish between continual damage caused by environmental exposures and senescence. In the latter, the evolutionary framework is based on the weakness of selection during the post reproductive lifespan, whereas accumulated damage can occur at any time during an individual's lifespan if the environmental exposure is sufficiently frequent. An increasing number of studies have also shown that even if malignancies do not necessarily lead to metastatic cancers, oncogenic phenomena in general (e.g., precancerous lesions, in situ carcinoma) are highly prevalent in animal populations and occur not just in post-reproductive individuals as previously believed [4]. This is also true in humans, as illustrated by several recent studies indicating that most, if not all, individuals harbor and accumulate

precancerous lesions and in situ tumors during their life in various organs (e.g., prostate, lung, thyroid, breast, pancreas) (see [5]). Another major reason for considering cancer as a disease that differs from a senescence problem is that the dynamics of malignant transformations and progression follow Darwinian principles. Somatic cellular selection and evolution are the fundamental processes leading to malignancy, with its many manifestations including immune system evasion, neoangiogenesis, metastasis, and resistance to therapies. In only a few months or years, these selective pro-cesses can favor the transformation of a single cell into a complexly organized collection of interacting cells (i.e., the solid tumor). Thus, although the initiation of cancers might have links with senescence-related processes, malignant progression itself relies on processes that differ from those directly linked to aging. Cancer is therefore not like most degenerative age-relative diseases (e.g., neurodegenerative diseases, several aspects of cardiovascular disease, macular degeneration, osteoporosis, sarcopenia), which are loss-of-function ailments. Rather, it is an example of a much smaller category of gain-of-function diseases (i.e., gain of cells, new cellular functions).

Also remarkably, eight naturally occurring transmissible contagious cancers [one lineage in dogs, two lineages in the Tasmanian devil (Sarcophilus harrisii), and five lineages in bivalves] have so far been recorded [6]. Tasmanian devil facial tumor disease (DFTD) illustrates how cancer can act as an evolutionary force. The recent epidemic of DFTD has caused a massive (>85\%) population decline in Tasmanian devils since the disease emerged in 1996 and is a significant selective force and a key threat to the long-term survival of this species.

Other cancers are not directly contagious but have (as in other chronic diseases; see [7]) infectious causations not related to senescence. These include Epstein- Barr virus, hepatitis B and C viruses, the bacterium Helicobacter pylori, human papilloma virus, and the trematodes Schistosoma haematobium, S. japonicum, and S. mansoni, which have been shown to be likely causal triggers of cancers of the lymph nodes, liver, stomach, cervix, bladder, colon, and liver, respectively (see [8]). In addition, the complete list of oncogenic pathogens is probably far from being fully known. Finally, it has long been known that in some cases a cancer can spontaneously regress and even disappear without treatment in both humans and animals. All of these features are not classical attributes of senescence pathologies, suggesting that cancer should thus be considered separately.

Why cancer has been predominantly viewed as a senescence pathology and thus been ignored or considered as noise by ecologists is due to at least two reasons: (i) an understandable focus on metastatic forms, which have obvious and serious impacts on the patient/host and usually occur late in life; and (ii) when performance in fitness-related traits varies between individuals in nonhuman animals, they are likely to be attributed to reasons other than malignancies, such as intraspecific variability, infectious diseases, or bad genes sensu lato. The reason for this is that cancer is not something that many ecologists consider as one of the many selective pressures acting on animals, although it is likely to be pervasive, and this may lead to individual differences in condition or performance. That is, a part of the variation in individual phenotypes is likely, at any time point, to be influenced by the state of the oncobiota (i. e., malignant cell communities) [9]. 
The importance of cancer (long before metastasis) in ecology and evolution is presently unknown despite it being likely to be highly relevant, since a reduction in body condition, even small, is usually associated with higher risk of predation and/or of infection, and reduced competitiveness/attractiveness in sexual selection processes in the wild [10]. Over half a billion years ago, multicellular organisms evolved several cancer suppression mechanisms (e.g., apoptosis, effective DNA repair, epigenetic modifications, telomere shortening, tissue architecture, immune surveillance). However, assuming that cancer, because of these protective mechanisms, is no longer a problem for reproducing animals is, at least in our opinion, a naive view. Cancer, like all diseases, is usually associated with tradeoffs at some level [11], and at least for this reason the mechanisms employed by hosts to cope with cancer cannot be considered in isolation from other functions that govern living organisms. More-over, recent work suggests that, in addition to resistance mechanisms to cancer, selection has also favored adjustment of life history traits and tolerance mechanisms [12]. Because these mechanisms allow hosts to alleviate the fitness costs of cancer without preventing its progression, this suggests that tumorbearing individuals in populations could be more frequent than currently predicted.

Although metastatic cancers primarily cause major pathological manifestations at later life stages in laboratory animals, we should not underestimate the adaptation-invoking role of this disease in shaping the ecology and evolution of animals throughout the lifespan. Also, even when invasive cancer is apparently absent in an organism, we cannot ignore the potential cost paid by this organism to maintain such a cancer-free status. It is time to adopt a novel perspective on cancer, especially its contribution to what evolutionary ecologists describe as interindividual variability [9]. Another reason for considering cancer is that most, if not all, ecosystems on our planet are now polluted by mutagenic substances to a greater extent than ever before, to an extent that the incidence of cancers in wildlife is likely to increase significantly in the near future. Directing our attention to the effects of noninvasive (sublethal) cancer should help to change the general concept of the impact of cancer on fitness. Currently the main limitation for scientists is methodology, primarily the lack of noninvasive diagnostic techniques to evaluate the oncobiotic state of individuals. Promising tools are, however, emerging (e.g., detection of circulating tumor cells or tumor DNA). Making the distinction between 'cancer' and 'malignancies' can help in understanding how the great majority of cancers occur in old age even while more common malignancies in youth can still impair fitness. After having acknowledged the importance of parasites and then microbiota, it is time to open the black box of oncobiota.

\section{Acknowledgments}

This work was supported by the ANR (Blanc project EVOCAN), the CNRS (INEE), an International Associated Laboratory Project France/Australia, the Montpellier Hérault Sport Club M.H.S.C, and André Hoffmann (Fondation MAVA).

References

1. Chaffer, C.L. and Weinberg, R.A. (2011) A perspective on cancer cell metastasis. Science 331, 1559-1564

2. Frank, S.A. (2004) Age-specific acceleration of cancer. Curr. Biol. 14, 242-246

3. Rozhok, A.I. and DeGregori, J. (2016) The evolution of lifespan and age-dependent cancer risk. Trends Cancer 2, 552-560

4. Madsen, B. et al. (2017) Cancer in the animal kingdom. In Ecology and Evolution of Cancer (Ujvari, B. et al., eds), pp. 11-46, Elsevier

5. Folkman, J. and Kalluri, R. (2004) Cancer without disease. Nature 427, 787-787

6. Ujvari, B. et al. (2017) Transmissible cancer: the evolution of interindividual metastasis. In Ecology and Evolution of Cancer (Ujvari, B. et al., eds), pp. 167179 , Elsevier

7. Ewald, P.W. Darwinian medicine: evolutionary approaches to disease. In The International Encyclopedia of Anthropology (Callan, H., ed.), John Wiley \& Sons (in press)

8. de Martel, C. and Franceschi, S. (2009) Infections and cancer: established associations and new hypotheses. Crit. Rev. Oncol. Hematol. 70, 183-194

9. Thomas, F. et al. (2017) The importance of cancer cells for animal evolutionary ecology. Nat. Ecol. Evol. 1, 1592-1595

10. Vittecoq, M. et al. (2013) Cancer: a missing link in ecosystem functioning? Trends Ecol. Evol. 28, 628-635

11. Jacqueline, C. et al. (2017) Cancer: a disease at the crossroads of trade-offs. Evol. Appl. 10, 215-225

12. Arnal, F. et al. (2017) Cancer brings forward oviposition in the fly Drosophila melanogaster. Ecol. Evol. 7, 272- 276 